\title{
Moving around the thymus: More technology, more safety, more efficiency
}

\author{
Tommaso Claudio Mineo, $\mathrm{MD},{ }^{\mathrm{a}}$ and Vincenzo Ambrogi, $\mathrm{MD}, \mathrm{PhD}^{\mathrm{b}}$
}

\footnotetext{
From the Departments of a Surgery and Experimental Medicine and ${ }^{\mathrm{b}}$ Thoracic Surgery, Tor Vergata Multidisciplinary Myasthenia Gravis Unit, Tor Vergata University, Rome, Italy.

Disclosures: Authors have nothing to disclose with regard to commercial support

Received for publication March 5, 2016; accepted for publication March 10, 2016; available ahead of print April $18,2016$.

Address for reprints: Tommaso Claudio Mineo, MD, Cattedra di Chirurgia Toracica, Policlinico Tor Vergata, Viale Oxford 81,00133 Rome, Italy (E-mail: mineo@med.uniroma2.it).

J Thorac Cardiovasc Surg 2016;152:280-1

$0022-5223 / \$ 36.00$

Copyright (c) 2016 by The American Association for Thoracic Surgery

http://dx.doi.org/10.1016/j.jtcvs.2016.03.045
}

Dr Yano and colleagues are to be congratulated for their initial experience with this technically advanced access to the neoplastic thymus. ${ }^{1}$ Over the past several decades, thymectomy has gained popularity as an effective treatment for myasthenia gravis (MG), thymoma, and other malignancies in the anterior mediastinum. Median sternotomy firmly remains the standard open approach to the thymus.

However, as a result of the advancement in the surgical technology, new less invasive approaches have progressively gained a role in the management of the nonthymomatous MG and small, noninvasive thymomas. ${ }^{3}$ These include transcervical surgery, video-assisted thoracic surgery (VATS), and robotic VATS.

Reduced operative trauma, less postoperative pain, shorter hospital stay, good cosmesis, and subsequent better postoperative quality of life are the most attractive features of these approaches. They have the requisite qualities requested by patients, surgeons, and neurologists, and also by economic administrators in terms of cost-benefit ratio.

Among the multiform approaches proposed, we like to emphasize the subxiphoid route. This access, combined with VATS, was introduced by our group for the palpation plus excision of lung metastases ${ }^{5}$ and subsequently popularized by Detterbeck and Egan. ${ }^{6}$ The subxiphoid incision was widely used as hybrid approach for thymectomy by Zielinski. $^{7}$ We found it to be easy and much less painful compared with the other transthoracic minimally invasive approaches to nonthymomatous thymectomy. More recently, novel technological advances have made it possible to perform these operations through a unique subxiphoid approach, which represents the latest challenge for the most experienced surgeons in VATS thymectomy. ${ }^{8}$

Ultimately, Yano and colleagues removed a neoplastic thymus through a technical, safe and effective uniportal subxiphoid approach. The success of this operation is based on 2 positive conditions. First, the authors performed a very detailed evaluation of the extension of the thymoma with a computed tomography scan of the mediastinum.

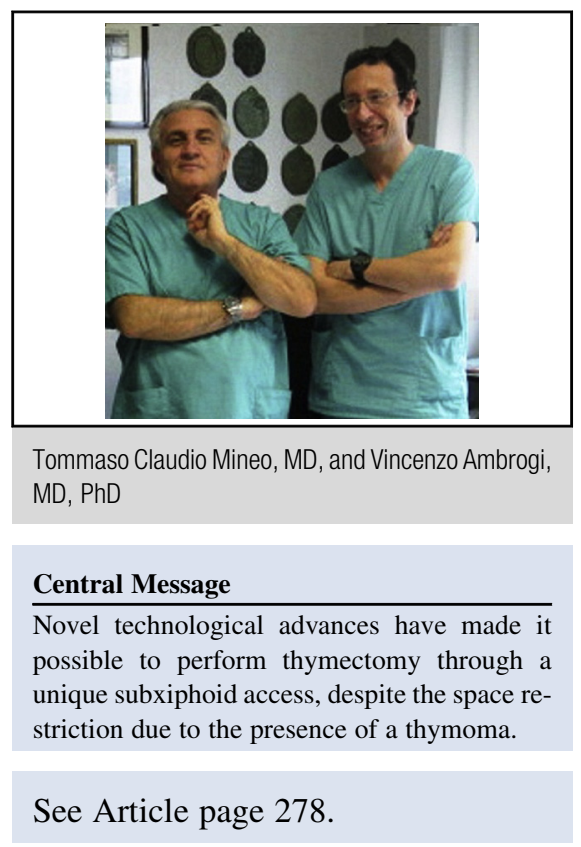

This assessment represents a pivotal precaution when performing surgery for a neoplastic thymus through a minimal or uniportal access. The study should exclude the presence of incidental vascular abnormalities, complex loco-regional anatomy, and involvement of adjacent structures. A safe vascular control is the main prerequisite for carrying out a thymectomy through the subxiphoid route. The prediction of neoplastic invasion of phrenic nerves, pericardium, and great vessels should suggest an open approach.

Second, the modern uniportal instrumentation can make the difference. The use of a long camera scope reduces interference between the surgeon's hands and the camera head. The development of angled instruments has made the operation more feasible. During the procedure, instruments are continuously exchanged to avoid interference and conflicts. The coordination and the position of these instruments allows surgeons to reach the "remote" cervical region, thus providing a satisfactory operative field for the identification of the anatomic structures in this area. Nevertheless, the intraoperative finding of adherences with the phrenic nerves and the great vessels, as well as the need to perform risky maneuvers, should induce the surgeon to convert the subxiphoid approach to a more comfortable access.

The use of this novel approach reopens the never-ending debate about the use of minimally invasive surgery for 
thymomas. Although VATS thymectomy has recently gained increasing acceptance for the treatment of Masaoka stage I noninvasive thymomas, some criticism about oncologic outcomes with a minimally invasive approach remains. The VATS approach to thymoma might occasionally result in spillage of neoplastic cells in the adjacent spaces and pleural cavities. On the other hand, no local or pleural disease recurrence for stage II thymoma resected by VATS was observed by Cheng et al. ${ }^{9}$ Similarly, in a large comparative study, Pennathur et $\mathrm{al}^{10}$ found no significant difference in disease recurrence for early-stage thymomas.

There is no doubt that an important factor that likely affects the success of the VATS approach is the dimension of the lesion. Thus, clinically diagnosed Masaoka stage I and II thymomas could be safely treated by VATS approach. Zhang et al ${ }^{11}$ have used a bilateral VATS approach in patients with Masaoka stage IIIa thymomas with sufficient results.

Imaging devices can accurately describe the position and the extension in up, down, and sideways orientations of the tumor, identifying the best route for approaching the resection. However, the surgeon should keep in mind that thymomas larger than $5 \mathrm{~cm}$, locally advanced, are difficult to manage because they further reduce the working mediastinal space.

We conclude with 2 observations. First, the thymus is attacked from all sides: from the front, top, bottom, and both sides, but nevertheless remains "patient and mysterious" in the depth of the mediastinum awaiting a new surgical assault. Second, the wide variety of the approaches developed thus far should be regulated by a simple statement: before you start, you should be sure about what are you looking for, and after you finish, you must confirm that you made the correct evaluation.

\section{References}

1. Yano M, Moriyama S, Haneda H, Nakaneshi R. Thymectomy using the subxiphoid approach. J Thorac Cardiovasc Surg. 2016;152:278-9.

2. Ambrogi V, Mineo TC. Active ectopic thymus predicts poor outcome after thymectomy in class III myasthenia gravis. J Thorac Cardiovasc Surg. 2012;143: 601-6.

3. Mineo TC, Ambrogi V. Left VATS thymectomy. In: Mineo TC, ed. Novel Challenges in Myasthenia Gravis. New York: Nova Science Publishers; 2015:415-49.

4. Ng CSH, Yim APC. Right VATS thymectomy: conventional and single-port approaches. In: Mineo TC, ed. Novel Challenges in Myasthenia Gravis. New York: Nova Science Publishers; 2015:395-414.

5. Mineo TC, Pompeo E, Ambrogi V, Pistolese C. Video-assisted approach for tranxiphoid bilateral pulmonary metastasectomy. Ann Thorac Surg. 1999;67: 1808-10.

6. Detterbeck FC, Egan TM. Thoracoscopic using a substernal handport for palpation. Ann Thorac Surg. 2004;78:1031-6.

7. Zielinski M. Alternative approaches to nonthymomatous thymectomy for myasthenia gravis through the subxiphoid incision. In: Mineo TC, ed. Novel Challenges in Myasthenia Gravis. New York: Nova Science Publishers; 2015:479-88.

8. Suda T. Single-port thymectomy using a subxiphoid approach: surgical technique. Ann Cardiothorac Surg. 2016;5:56-8.

9. Cheng YJ, Kao EL, Chou SH. Videothoracoscopic resection of stage II thymoma: prospective comparison of the results between thoracoscopy and open methods. Chest. 2005;128:3010-2.

10. Pennathur A, Qureshi I, Schuchert MJ, Dhupar R, Ferson PF, Gooding WE, et al Comparison of surgical techniques for early-stage thymoma: feasibility of minimally invasive thymectomy and comparison with open resection. J Thorac Cardiovasc Surg. 2011;141:694-701.

11. Zhang G, Li W, Chai Y, Wu M, Zhao B, Fan J, et al. Bilateral video-assisted thoracoscopic thymectomy for Masaoka stage IIIa thymomas. Thorac Cardiovasc Surg. 2015;63:206-11. 